\title{
EasyDSP: Problem-Based Learning in Digital Signal Processing
}

\author{
Kaveh Malakuti and Alexandra Branzan Albu \\ Department of Electrical and Computer Engineering \\ University of Victoria (BC) Canada \\ malakuti@ece.uvic.ca,aalbu@ece.uvic.ca
}

\begin{abstract}
This paper presents a pilot pedagogical project that combined for the first time problem-based learning $(P B L)$ with a lecture-based instructional style in Digital Signal Processing I. This new course format was offered during the Spring 2008 term at the University of Victoria, Canada. The PBL component was implemented via EasyDSP, a custom-designed framework for system design and signal analysis.
\end{abstract}

\section{Introduction}

Learning core concepts in signal processing courses is a challenge for undergraduate students. One possible cause for this is the gap between understanding the mathematical formalism of such concepts and being able to use them for solving real-world problems.

To bridge this gap, instructional formats other than purely lecture-based need to be investigated. For instance, Buck and Wage [1] show that 'students learn core concepts in signal processing better when the class requires active participation than when a traditional lecture format is used.' They employ an instructional technique called Active and Cooperative Learning (ACL) which combines in-class 'readiness' quizzes with in-class problem-solving exercises in order to reinforce important concepts. The ACL method is proven in [1] to enhance the learning outcomes of signal processing at a conceptual level, thus getting similar results to those reported in [2, 3] for theoretical physics courses.

Our belief is that signal processing is an applied engineering field, although it relies heavily on mathematical concepts. Therefore, we investigate whether Problem-Based Learning (PBL) in combination with a lecture-based teaching style is able to enhance the learning outcomes of an introductory core course in digital signal processing. This paper reports on a pilot pedagogical project that combined for the first time problem-based learning with a lecture-based instructional style in Digital Signal Processing I. This new course format was offered during the Spring 2008 term at the University of Victoria, Canada.

It is important to mention that our implementation of problem-based learning is different from the experiential learning, or the learning-by-doing approach that is usually implemented in engineering classes via laboratory assignments. The problems to be solved in such assignments are specified in closed form by the course instructor, and they follow closely the theoretical content of the lectures. Our initiative aimed at providing a framework for solving multimedia-related DSP problems, where students were encouraged to formulate their own problems. More details are to be found in section 2 .

The remainder of the paper is structured as follows. Section 2 describes the PBL component that was added to the lecture-centric course. Section 3 provides an overview of the custom-designed framework for system design and analysis. Section 4 discusses the lessons that we learned during the first offering of this new course format and outlines future work directions.

\section{Problem-based Learning for DSP I}

This section describes the proposed PBL component in the context of the Digital Signal Processing course and discusses the relevance of this component for the learning outcomes of the course. Contextual information about the course content is presented first.

\subsection{Course Overview}

Digital Signal Processing I is a core course in electrical engineering curricula. The concepts and techniques that form the theoretical foundation of the course are of great importance to many engineering disciplines. Oppenheim and Willsky [4] write that "the scope of potential and actual applications of the methods of signal and system analysis continues to expand as 
engineers are confronted with new challenges involving the synthesis and analysis of complex processes.”

As a result of taking the DSP 1 course at UVic, students should be able to:

- Master fundamental concepts such as linearity, time-invariance, impulse response, convolution, frequency response, Ztransform, Discrete Time Fourier Transform, sampling, and digital filter design as applied to DSP.

- Understand the applicability of digital signal processing (DSP) techniques to various fields of electrical engineering and specifically to multimedia applications.

Our focus on multimedia applications of DSP is motivated by their ubiquitous nature in our information society. Multimedia-related DSP problems formulated in lay terms are likely to trigger more interest from students and thus have the potential to help them integrate theoretical knowledge with problem solving skills.

\subsection{Problem-Based Learning Component}

Problem-based learning is becoming increasingly popular in engineering. It is typically implemented via engineering design projects, as shown in [5-7]. Projects represent the hands-on component of engineering courses, where students apply theoretical knowledge in the context of a specific application. However, formulating an appropriate project for an introductory DSP course presents challenges on how to avoid coursework overload. DSP I is very dense from a theoretical viewpoint and thus requires a fair amount of effort from students towards mastering the concepts and techniques taught in the course. The PBL component needs to be carefully crafted in order to complement well the theoretical course content without overloading the student.

Our hypothesis is that while observing how simple DSP components (e.g. $1^{\text {st }}$ or $2^{\text {nd }}$ order filters, delays and up/down samplers) work on familiar signals students will grasp more easily fundamental concepts such as superposition, convolution, frequency response, sampling etc. The programming environment (see Section 3) enabled the direct perception of how filters change the input signals.

The projects were formulated so that the students had to achieve a well-defined goal. This goal was reachable via multiple paths, i.e. by choosing among several multi-step signal processing approaches. Two example problems were proposed by the instructor. Students were encouraged to specify their own problem within the area of audio/image processing, in a similar way to the two example problems which are outlined below.

\section{A) Enhancement of speech signal}

Speech signals are recorded using a microphone with unshielded cable. As a result, the signals are contaminated with $60 \mathrm{~Hz}$ interference. Also, due to the fact that the microphone's frequency response is not flat and that higher pitches are suppressed, the recorded voice sounds humming, with a background buzz.

Students are asked to design a DSP system to enhance the signal-to-noise ratio of their speech signal and to modify its spectrum in such a way that it sounds as natural as possible.

In the planning stage, students need to select their signal processing blocks and to justify their selection. The implementation stage involves the interconnection of the blocks and the parametric adjustment of each block.

\section{B) Enhancement of print-out quality of facial images}

Facial images are captured using a low quality webcam. The images feature Gaussian noise and are over-exposed, due to excessive lighting. The quality of the images needs to be optimized for printing purposes.

The planning stage needs to identify the processes to be performed (such as de-noising, contour sharpening, histogram equalization, removal of specular regions etc.). The implementation stage involves interconnecting the processes above, as well as tuning their parameters.

All problems (either instructor- or student-formulated) were to be solved using EasyDSP, a custom-designed programming environment, detailed in Section 3.

The project deliverables consisted of:

- A written report describing the problem, its solution, and the design methodology.

- The complete, functional system saved in EasyDSP format so that it could be tested for marking purposes.

- All signals used in system design and testing.

The project counted $5 \%$ of the total course mark, with the possibility of gaining up to $5 \%$ extra points for high-quality projects dealing with creative problems. 


\section{EasyDSP: a custom-designed framework for PBL}

The main goal of the EasyDSP environment is to enable a fast and easy prototyping/design of DSP systems for image and audio processing. The paradigm that we propose is direct visual manipulation of discrete signals and systems. It is similar to the WYSIWYG (What You See Is What You Get) paradigm, a very popular choice for text editors.

The graphical interface of EasyDSP allowed students to manipulate the parameters of each incorporated block for digital signal processing and thus to gain understanding of how it affects the input signals.

The development of a system in EasyDSP consists of the following steps:

- Loading or creating the input signals

- Creating filters

- Interconnecting sources, filters and arithmetic blocks

- $\quad$ Placing probes at desired points in the system for signal investigation (Sink)

- System simulation

- Viewing and saving the outputs (Sink)

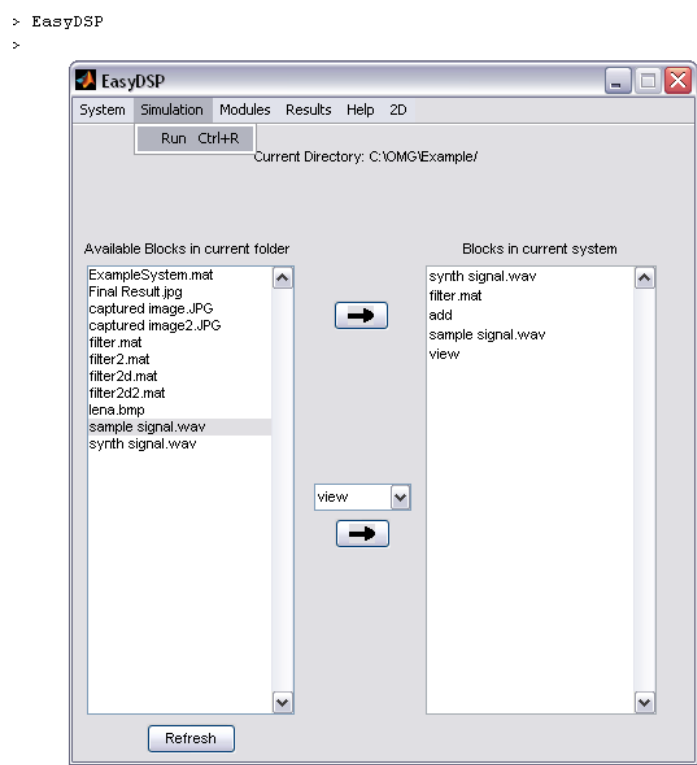

Figure 1. Main user interface for system design and simulation in EasyDSP
Figure 1 shows the main user interface in EasyDSP. The left column contains all blocks (either prebuilt or created by user) available for system development. The right column contains the blocks of the system under development.

Filter design has been implemented via dropdown menus and sliders for choosing filter type and parameters (see Figure 2). Once the filter is designed, it can be visualized using a pop-up window as shown in Figure 2b. Students can investigate magnitude, phase, impulse and unit step responses of their filter as well as zero-pole, group delay and phase delay plots. These filter design options make EasyDSP more intuitive than Matlab's FDATOOL, since they enable novice users to locate, manipulate, and visualize their filters faster, without getting lost in advanced options.

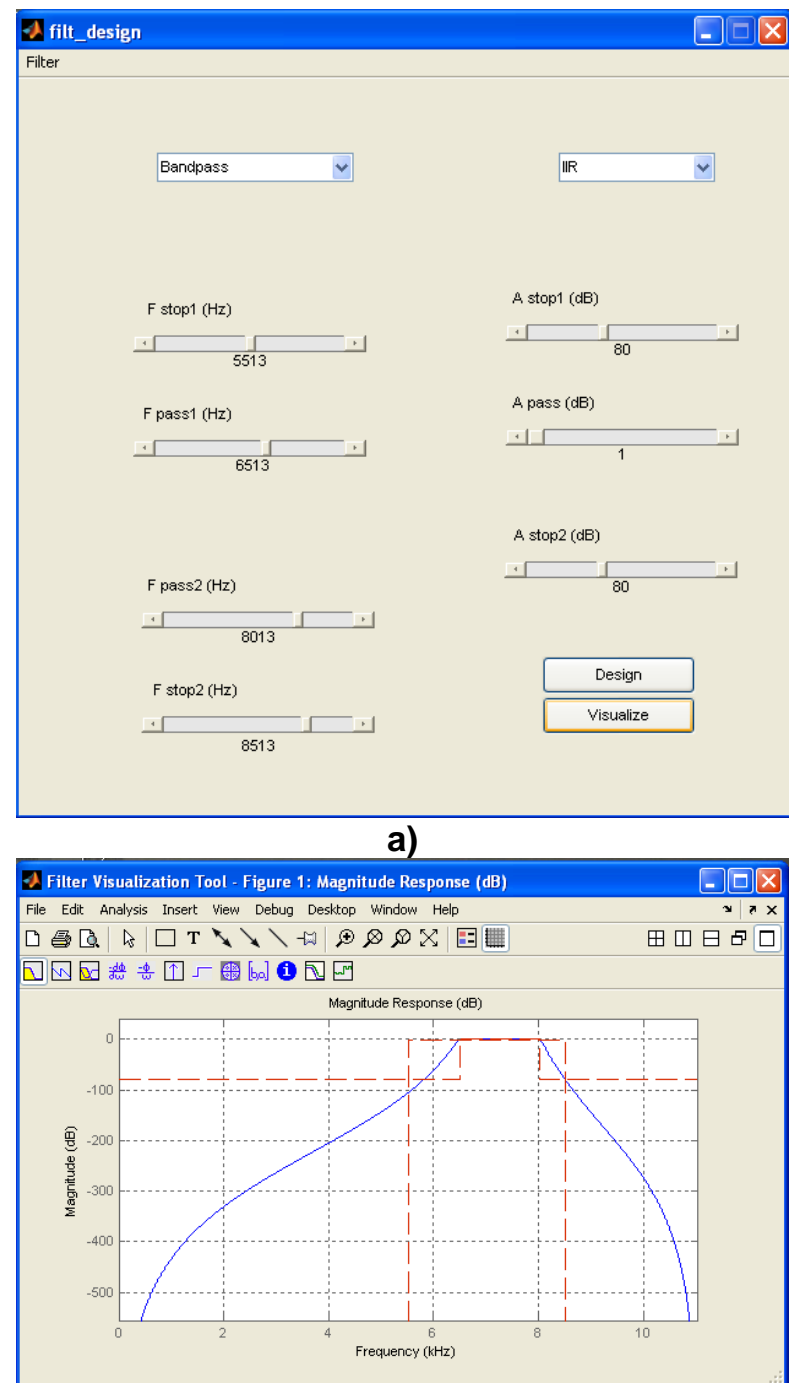

b)

Figure 2. a) Filter design; b) Filter visualization in EasyDsp 


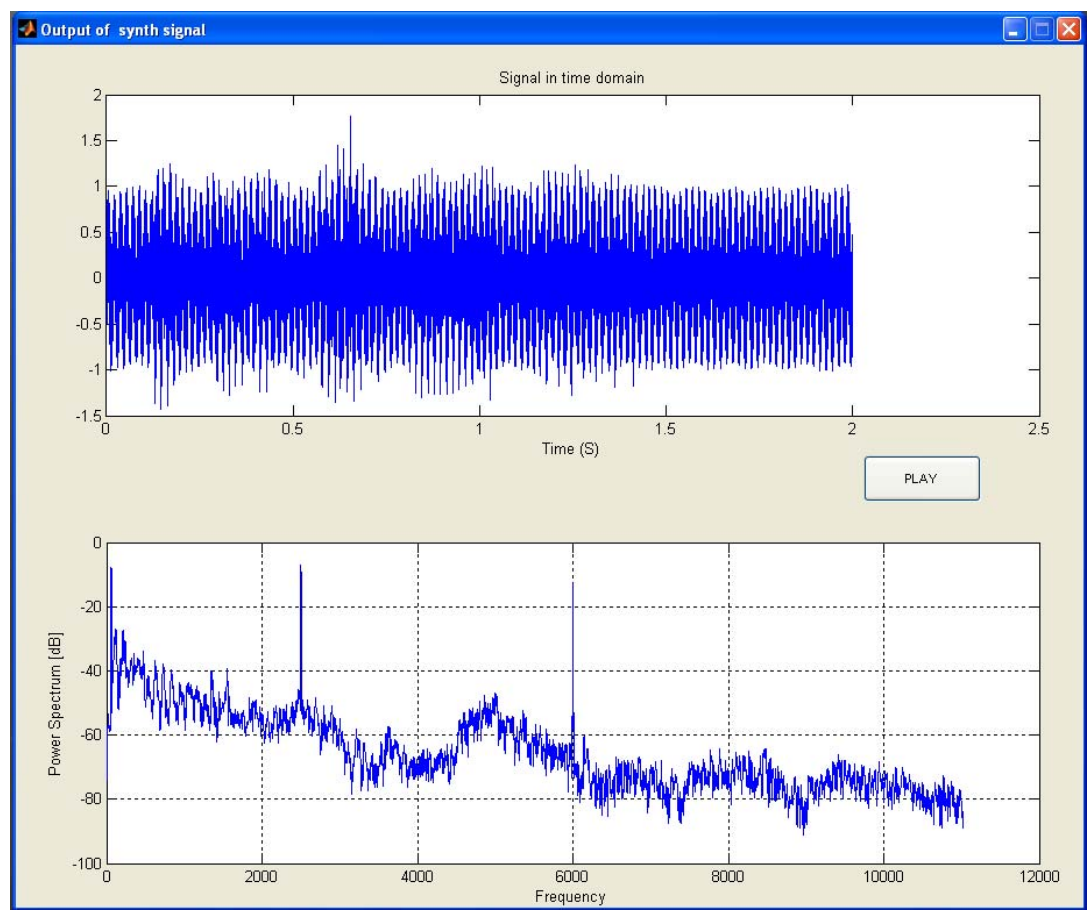

Figure 3. Simultaneous time- and frequency-domain visualization in EasyDSP

As shown in Figure 3, EasyDSP also provides the option to probe the signal at any desired location in the system by using pop-up windows or by saving it to a file. Probes are considered a signal sink and can be placed at the output of any block on the system. The simultaneous time (for audio signals)/space (for images) and frequency-domain visualization is useful for understanding the correlation between time(space-) and frequency domain representations, as well as the trade-off between different constraints imposed on filters in either time, space or frequency.

\section{Lessons learned and future work}

The first offering of this new hybrid course format has been an intense learning experience for the instructional team. Feedback from students was collected via anonymous surveys and course evaluation forms. At present, the results of a formal evaluation are pending ethics approval. This is why we will present only qualitative observations that will enable us to improve the quality of the PBL component for DSP I in future course offerings.

The challenging aspect of adopting the PBL style for large classes and introductory core courses has been confirmed by our experience. Our class had over 70 students enrolled, and the instructional resources for the PBL component were limited to one teaching assistant and the course instructor. Along with direct meetings, on-line live help was made available to students for their projects. However, this feature was used by less than $50 \%$ of the enrolled students. We attribute this low popularity of on-line live help to the fact that this feature is not used for other ECE courses at UVic, and students need time to adopt it.

Student-formulated problems were found in approximately $15 \%$ of the total number of handed-in projects. Interesting applications included image segmentation via edge detection, creating audio effects via digital filtering, and segmentation of piano sounds based on harmony analysis.

The low percentage of student-formulated problems is not surprising, since DSP I students had little exposure to creative problem solving assignments prior to taking our course. Also, some student comments expressed the desire to receive a more structured project assignment formulated in closed-form. Efforts will be made to reverse this preference about passive, directed learning in order to stimulate students' creativity, accountability and involvement in project work. Such efforts will include more in-class project-related discussions and brainstorming sessions. 
The EasyDSP software needs improvement in terms of stability, flexibility, and efficiency for the implementation of user-formulated filters. To help students become familiar with EasyDSP, the improved software will also be used for in-class demonstrations and discussions that will complement the lecture content. This will provide a common element between the lecture-based course content and its PBL component.

\section{Acknowledgments}

We acknowledge the financial support the Design Engineering Office at UVic via a Curriculum Development Grant.

\section{References}

[1] J. R. Buck and K.E. Wage. "Active and Cooperative Learning in Signal Processing Courses”, IEEE Signal Processing Magazine, vol. 22(2), March 2005, pp. 76-81.

[2] R.R. Hake, "Interactive-engagement vs. traditional methods: A six-thousand-student survey of mechanics test data for introductory physics courses,” Am. J. Phys., vol. 66, pp. 64-74, 1998.

[3] C.H. Crouch and E. Mazur, "Peer instruction: Ten years of experience and results,” Am. J. Phys., vol. 69, pp. 970977, 2001.

[4] A. V. Oppenheim and A. S. Willsky. Signals and Systems. Prentice Hall, 2nd edition, New Jersey, USA, 1997.

[5] M.L. Ho, A.B. Rad, and P.T. Chan, "Project-based learning”, IEEE Control Systems Magazine, Oct. 2004, pp. 88-91.

[6] D. Davenport, "Experience using a project-based approach in an introductory programming course", IEEE Transactions on Education, vol. 43(4), 2000, pp. 443-448.

[7] J. Macias-Guarasa, J.M. Montero, R. San-Segundo, A. Araujo, and O. Nieto-Taladriz, "A Project-Based Learning Approach to Design Electronic Systems Curricula”, IEEE Transactions on Education, 49 (3): 389-397, 2006. 